\title{
Bioprótese aórtica porcina "Stentless": acompanhamento clínico a médio prazo
}

Mário O. VRANDECIC* ${ }^{*}$ Bayard GONTIJO FILHO*, Fernando Antônio FANTINI*, João Alfredo PAULA E SILVA*, Ozanan C. OLIVEIRA* ${ }^{*}$, Idail Costa MARTINS Jr. ${ }^{\star}$, Juscelino Teixeira BARBOSA ${ }^{*}$, Roberto M. OLIVEIRA*, Sandra O. S. AVELAR*, Hebert C. MIOTTO*, Mauricio R. BARBOSA*, Érika VRANDECIC*, Ektor VRANDECIC*

RBCCV 44205-232

VRANDECIC, M. O.; GONTIJO FILHO, B.; FANTINI, F. A.; PAULA E SILVA, J. A.; OLIVEIRA, O. C.; MARTINS Jr., I. C.; BARBOSA, J. T.; OLIVEIRA, R. M.; AVELAR, S. O. S.; MIOTTO, H. C.; BARBOSA M. R.; VRANDECIC, E.; VRANDECIC, E. - Bioprótese aórtica porcina "Stentless": acompanhamento clínico a médio prazo. Rev. Bras. Cir. Cardiovasc., 9 (1): 60-63, 1994.

RESUMO: O presente trabalho visa apresentar os resultados obtidos a médio prazo com o uso da prótese valvar aórtica porcina sem suporte "Stentless" Biocor. De maio de 1990 a dezembro de 1993, 120 pacientes foram submetidos à troca de valva aórtica usando-se a prótese "Stentless", no Biocor Instituto de Belo Horizonte, Brasil. A idade dos pacientes variou de 11 a 76 anos (média: 36 anos), sendo 85 com menos de 40 anos. O sexo predominante foi o masculino com $69 \%$ dos casos. A seqüela de cardite reumática foi a etiologia mais freqūente ( 64 casos), seguida da disfunção de prótese aórtica (20), estenose aórtica congênita (11), endocardite em prótese aórtica (10), endocardite em valva aórtica (6), degeneração mixomatosa (5) e calcificação senil (4 casos). A técnica cirúrgica incluiu o uso de circulação extracorpórea com hipotermia moderada, cardioplegia cristalóide e aortotomia transversa dirigida para o seio de Valsalva nāo coronariano. A bioprótese foi implantada usando-se sutura contínua, interrompida, ou uma combinação das duas. A aortotomia foi ampliada com retalho de pericárdio bovino em 47 pacientes e outros procedimentos associados foram empregados em 30 casos. A mortalidade hospitalar foi de $5 \%$. Quatorze (11,6\%) pacientes apresentaram complicaçōes pós-operatórias, a maioria sem maiores problemas. No entanto, $5(4,1 \%)$ pacientes, operados no início da experiência, tiveram de ser submetidos a implante de marcapasso definitivo. Ocorreram seis óbitos tardios, todos não relacionados à bioprótese. Foram necessários quatro reoperações tardias, duas para correção de deiscência da sutura inferior e duas devido a endocardite bioprótese implantada. Estudos ecocardiográficos seriados realizados ao longo do seguimento tardio mostram função adequada com gradientes desprezíveis em todos os pacientes. A bioprótese encontra-se suficiente em $95.3 \%$ dos casos e nos demais, pequenos jatos de regurgitaçăo sem significado hemodinâmico podem ser detectados. Concluímos que, a médio prazo, a bioprótese aórtica sem suporte, Biocor apresentou ótimos resultados clínicos e excelente desempenho hemodinâmico. Um seguimento mais longo ainda é necessário.

DESCRITORES: próteses cardíacas artificiais, aórticas.

\section{INTRODUÇÃO}

Os bons resultados clínicos com o uso de homoenxertos em posição aórtica levou ao desenvolvimento de novos caminhos para a cirurgia de troca da valva aórtica. O princípio do uso de tecidos cardíacos anatomicamente idênticos é conhecido como "conceito Stentless". Esta idéia representa o caminho mais natural para o tratamento das valvas cardíacas alteradas. Uma das vantagens desta nova prótese biológica é a completa ausência do anel rígido, anel que sabidamente aumenta o estresse mecânico nos tecidos, reduzindo a durabilidade. Este novo desenho atômico proporciona assim melhor função valvar.

Trabalho realizado no Biocor Instituto, Belo Horizonte, MG, Brasil.

Apresentado ao $21^{\circ}$ Congresso Nacional de Cirurgia Cardiaca. Porto Alegre, RS, 24 a 25 de março, 1994.

- Do Biocor Hospital, Belo Horizonte, MG

Endereço para correspondência: Mário Vrandecic, Caixa Postal 106 CEP 30161-970, Belo Horizonte, MG, Brasil. 
VRANDECIC, M. O.; GONTIJO FILHO, B.; FANTINI, F. A.; PAULA E SILVA, J. A.; OLIVEIRA, O. C.; MARTINS Jr., I. C.; BARBOSA, J. T.; OLIVEIRA, R. M.; AVELAR, S. O. S.; MIOTTO, H. C.; BARBOSA, M. R.; VRANDECIC, E.; VRANDECIC, E. - Bioprótese aórtica porcina "Stentless": acompanhamento clínico a médio prazo. Rev. Bras. Cir. Cardiovasc., 9 (1): 60-63, 1994.

Desde que as valvas cardíacas têm funções específicas e suportam diferenças de pressão, o desenho e a forma devem ser respeitados para atingir a proposição de boa função e maior durabilidade.

A bioprótese aórtica porcina "Stentless" Biocor (BAPSB)* é montada usando-se válvulas de valvas porcinas selecionadas, suturadas em um enxerto tubular de pericárdio bovino respeitando o formato ondulado da valva aórtica, com uma aba superior e inferior que permite a sua sutura na aorta.

Este trabalho mostra o acompanhamento clínico a médio prazo de pacientes nos quais a bioprótese porcina aórtica "Stentless" Biocor foi implantada.

\section{CASUÍSTICA E MÉTODOS}

1) Pacientes: Após aprovação ética, o estudo clínico teve início em maio de 1990. O consentimento foi obtido de todos os pacientes.

Cento e vinte pacientes foram submetidos a troca aórtica usando BAPSB.

Este grupo incluía 83 homens e 37 mulheres. A idade variou de 11 a 76 anos com média de 36 e 85 pacientes abaixo de 40 anos (Gráfico 1). A indicação para troca aórtica foi seqüela de doença reumática em 64 pacientes, valva aórtica bicúspide em 11 , endocardite em 6 , degeneração de valva aórtica em 5 e calcificação senil em 4 . Houve 30 reoperações nas quais 20 devido a falência tecidual protética e em 10 devido a endocardite na prótese (Tabela 1). Trinta e três pacientes tinham anéis aórticos calcificados, estreitados ou infectados.

2) Cirurgia: A técnica cirúrgica já foi discutida anteriormente 2, 6-8. Foram realizados 77 procedimentos associados: alargamento da aorta ascendente em 47, troca mitral em 15, troca mitral (reoperação) em 4, plastia mitral em 4 , cirurgia de revascularização do miocárdio em 5 e em 2 comissurotomia mitral (Tabela 2).

\section{GRÁFICO 1}

DADOS CLINICOS - N. 120

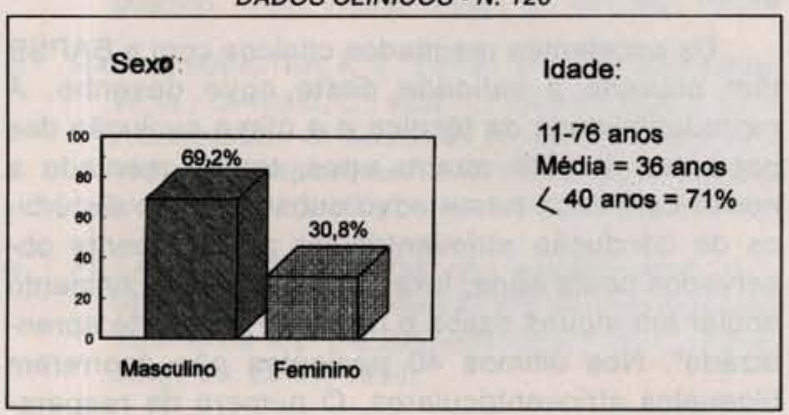

TABELA 1

ETIOLOGIA

\begin{tabular}{lc}
\hline - Doença reumática & $64(53.3 \%)$ \\
- Falência Protética & $30(25.0 \%)$ \\
Falência Primária & 20 \\
Endocardite Protética & 10 \\
- Endocardite Aórtica & $6(5.0 \%)$ \\
- Doença Aórtica Degenerativa & $5(4.2 \%)$ \\
- Valva Aórtica Bivalvulada & $11(9.2 \%)$ \\
- Calcificação Senil & $4(3.3 \%)$ \\
\hline
\end{tabular}

TABELA 2

PROCEDIMENTOS ASSOCIADOS N 77

\begin{tabular}{lc}
\hline Alargamento da Aorta Ascendente & $47(61 \%)$ \\
Troca Mitral & $15(20 \%)$ \\
Troca Mitral (reoperação) & $4(5 \%)$ \\
Plastia Mitral & $4(5 \%)$ \\
Revascularização do Miocárdio & $5(6 \%)$ \\
Comissurotomia Mitral & $2(3 \%)$ \\
\hline
\end{tabular}

3) Acompanhamento dos pacientes: Todos os pacientes foram acompanhados em intervalos de 3 meses. O acompanhamento incluia exame clínico, Eco bidimensional e Doppler-color e exames de sangue de rotina.

\section{RESULTADOS}

1) Houve, seis óbitos hospitalares, nenhum relacionado a bioprótese. Em 2 pacientes foi devido a falência orgânica progressiva devido ao péssimo estado pré-operatório e em 3 decorrente de arritmias (taquicardia ventricular em 2 e disfunção de marcapasso (provisório em um). Mediastinite foi a causa do óbito do $6^{2}$ paciente (Staphylococcus aureus) (Tabela 3).

Complicações hospitalares ocorreram em 14 pacientes. Nove desenvolveram bloqueio atrioventricular nos quais 4 retornaram a ritmo sinual regular e em 5 foi necessário marcapasso permanente. Revisão de hemostasia foi necessária em 4 pacientes e em 1 foi registrado AVC secundário, provavelmente por embolia aérea durante CEC. Todos os 14

TABELA 3

RESULTADOS

\begin{tabular}{ll}
\hline Mortalidade Hospitalar & $6(5,0 \%)^{*}$ \\
Mortalidade Tardia & $6(5,2 \%)^{*}$ \\
Reoperações & $4(3,6 \%)$ \\
Vazamento Paravalvar & 2 \\
$\quad$ Endocardite & 2 \\
"Não relacionado às válvulas & \\
\hline
\end{tabular}


VRANDECIC, M. O.; GONTIJO FILHO, B.; FANTINI, F. A.; PAULA E SILVA, J. A.; OLIVEIRA, O. C.; MARTINS Jr., I. C.; BARBOSA, J. T.; OLIVEIRA, R. M.; AVELAR, S. O. S.; MIOTTO, H. C.; BARBOSA, M. R.; VRANDECIC, E.; VRANDECIC, E: - Bioprótese aórtica porcina "Stentless": acompanhamento clínico a médio prazo. Rev. Bras. Cir. Cardiovasc., 9 (1): 60-63, 1994.

pacientes se recuperaram bem das complicações e receberam alta hospitalar.

Ocorreram seis óbitos tardios em 114 pacientes: em 1 devido a carcinoma pancreático, 3 meses após a operação e outro devido a endocardite fúngica (cândida albicans) no $2^{\circ}$ mês de pós-operatório; o terceiro paciente recebeu uma "bolada" na região de implante do maracapasso definitivo em um jogo de futebol, 6 meses após a operação e o quarto paciente faleceu em decorrência de embolia pulmonar fulminante, 6 meses após a operação. Em 2 pacientes não foi possível determinar a causa do óbito, mas as próteses encontravam-se inalteradas no estudo post-morten (arritmia cardiaca ?). Este estudo foi obtido em quatro dos óbitos tardios (Tabela 3 ).

2) Reoperação: Houve quatro reoperações no grupo aórtico, em dois decorrente de endocardite na prótese em pacientes primariamente operadas na fase aguda de endocardite infecciosa e nos outros dois devido a deiscência parcial da sutura anular, sutura contínua em anel aórtico calcificado. (Tabela 3). Todos os quatro reoperados tiveram a valva trocada com prótese BIOCOR "standart" No-REACT TM e se recuperaram bem da segunda troca. Cento e um $(90 \%)$ pacientes estão em classe funcional I e $9(7 \%)$ em classe II. Somente $2(3 \%)$ encontram-se em classe III (Gráfico 2).

3) Acompanhamento a médio prazo: Seguimento tardio foi obtido em 106 pacientes. Exames clínicos e laboratoriais não revelaram alterações dignas de nota. Ecodopplercardiograma demonstrou prótese aórtica "Stentless" suficiente em 97(92\%) pacientes. Em $9(8 \%)$ pacientes um pequeno jato pôde ser detectado pelo Eco, porém sem causar repercussão hemodinâmica significativa. Sessenta e quatro por cento das biopróteses "Stentless" foram de diâmetro 23 ou 25 , tamanho 21 representou $8 \%$,

\section{GRÁFICO 2}

PRÉ-OPERATÓRIO E PÓS-OPERATÓRIO-CLASSE FUNCIONAL (N.Y.H.A.)

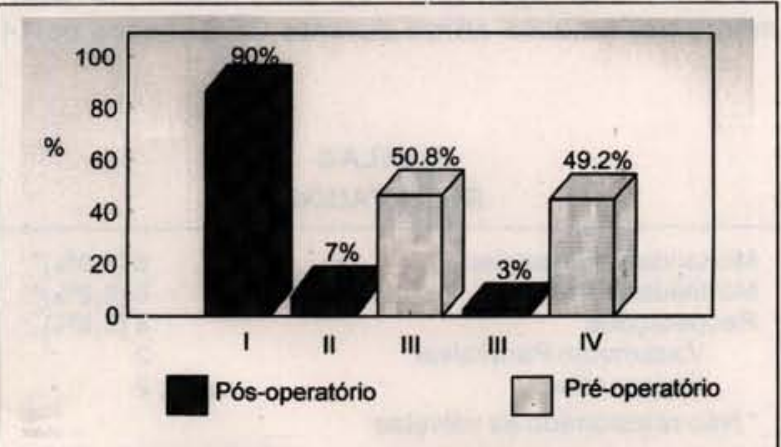

tamanho $27(26 \%)$ e tamanho $29(2 \%)$ dos casos. O gradiente de pico variou de 10 a $24 \mathrm{mmHg}$ e o gradiente médio foi de $10,5 \mathrm{mmHg}$ (Tabela 4). Cinco pacientes foram submetidos a cateterismo cardíaco que revelou próteses suficientes, boa função ventricular e nenhum gradiente.

A bioprótese "Stentless" aórtica manteve-se suficiente neste seguimento. Os jatos regurgitantes triviais observados à ecocardiografia desapareceram ou persistiram sem repercussão hemodinâmica.

TABELA 4

ANÁLISEECODOPPLERCARDIOGRÁFICA

\begin{tabular}{ll} 
Tamanho da Valva "Stentless" & $23-25 \mathrm{~mm}$ \\
V. maxm/sec & $2.3-2,9$ \\
Gradiente de Pico & $6-24 \mathrm{mmHg}$ \\
Gradiente Médio & $10,5 \mathrm{mmHg}$ \\
Fração de Ejeção V.E. & $55 \%-60 \%$ \\
Insuficiência Aórtica & \\
Nenhuma & $97(92 \%)$ \\
Leve & $9(08 \%)$ \\
\hline
\end{tabular}

\section{COMENTÁRIOS}

Em recente publicação, HAMMERMEISTER et alii $^{3}$, demonstraram sobrevida e índices de complicações similares entre próteses mecânicas e biológicas, e mostraram que nenhum modelo atual pode ser considerado como a prótese ideal. A pesquisa para o desenvolvimento de próteses com melhor desempenho e mais duráveis deve, portanto, continuar ${ }^{5}$. O comportamento a longo prazo de homoenxertos aórticos é encorajador; embora a manutenção de estoques com todos os tamanhos seja difícil e a insuficiência valvar tardia tenha sido observada, este é o modelo que naturalmente mais se aproxima do desejado 1,4 . A BAPSB foi desenvolvida para se aliar às condições anatômicas e à disponibilidade de tamanho, evitando-se insuficiência valvar tardia e superando as dificuldades técnicas encontradas com o uso de homoenxertos em geral.

Os excelentes resultados clínicos com a BAPSB têm provado a validade deste novo desenho. A reprodutibilidade da técnica e a ótima evolução dos pacientes em até quatro anos, tem aumentado a nossa confiança neste novo substituto. Os distúrbios de condução atrioventricular precocemente observados nesta série, foram reflexo do envolvimento anular em alguns casos e da usual "curva de aprendizado". Nos últimos 40 pacientes não ocorreram bloqueios atrioventriculares. $\mathrm{O}$ número de reopera- 
VRANDECIC, M. O.; GONTIJO FILHO, B.; FANTINI, F. A.; PAULA E SILVA, J. A.; OLIVEIRA, O. C.; MARTINS Jr., I. C.; BARBOSA, J. T.; OLIVEIRA, R. M.; AVELAR, S. O. S.; MIOTTO, H. C.; BARBOSA, M. R.; VRANDECIC, E.; VRANDECIC, E. - Bioprótese aórtica porcina "Stentless": acompanhamento clínico a médio prazo. Rev. Bras. Cir. Cardiovasc., 9 (1): 60-63, 1994.

ções (4) das quais duas foram secundárias a endocárdite recorrente, reflete o pobre nível sócio-econômico dos nossos pacientes. As deiscências de sutura que resultaram em reoperação, nos deram a oportunidade de aprender a escolher a melhor técnica de sutura (contínua ou separada dependendo do tipo de anel aórtico).
Os resultados obtidos, confirmados por estudos ecocardiográficos e de cateterismo cardíaco, consolidaram nossa fé neste procedimento e no novo desenho. Estudos randomizados estão sendo conduzidos para determinar a evolução a longo prazo, quando comparadas com as biopróteses convencionais.

VRANDECIC, M. O.; GONTIJO FILHO, B.; FANTINI, F. A.; PAULA E SILVA, J. A.; OLIVEIRA, O. C.; MARTINS Jr., I. C.; BARBOSA, J. T.; OLIVEIRA, R. M.; AVELAR, S. O. S.; MIOTTO, H. C.; BARBOSA, M. R.; VRANDECIC, E.; VRANDECIC, E. - Porcine Stentless heart valve substitutes: technical and clinical mid-term follow-up. Rev. Bras. Cir. Cardiovasc., 9 (1): 60-63, 1994.

ABSTRACT: From May 1990 to January 1994, one hundred and twenty (120) patients underwent aortic valve replacement with the use of the porcine aortic stentless valve. This group comprised of eighty-three (83) male and thirty-seven (37) female patients. The age ranged from eleven (11) to seventy-six (76) years with a mean of 36 . There were 85 patients under 40 years of age. Sixty-four patients (64) underwent their first aortic valve replacement due to rheumatic heart disease, thirty $(30)$ because of prosthetic valve failure and of those: twenty $(20)$ due primary tissue failure and in ten (10) due to prosthetic endocarditis, native aortic bicuspid valve in eleven (11) and senile calcificant aortic valve disease in four (4). Thirty-three (33) patients had aortic annular related pathology. The functional class revealed sixty-one (61) patients in class III and fifty-nine (59) in class IV. The longest follow-up was 42 months with a mean of 26 . The surgical technique used in the aortic valve surgery rendered consistent and reproducible results. The hospital mortality was $5 \%$ (6 patients). This mortality was not valve related. The hospital morbidity revealed full recovery of all patients. There were four (4) late reoperations, in two (2) due to recurrent endocarditis and in two (2) because of paravalvar leak. All patients had full recovery after the reoperation. There was a late mortality of six (6) patients (non valve related). The follow-up of these patients revealed full competent valve in ninety-seven (97) patients and only minor jet in nine (9). Most patients are in functional class I and II. The stentless concept has proven to be outstanding in the aortic position throughout the current follow-up. Although the test of time is required, we have gained confidence in the procedure due to the good clinical results.

DESCRIPTORS: heart valves prostheses, aortic.

\section{REFERÊNCIAS BIBLIOGRÁFICAS}

1 ANGELL, W. W.; ANGELL, J. D.; OUR, J. H. - Long term follow-up of viable frozen aortic homograft. $J$. Thorac. Cardiovasc. Surg., 93: 815-822, 1987.

CASABONA, R.; MOREA, M. \& VRANDECIC, M. P. Stentless porcine and pericarial valve in aortic position. Ann. Thorac. Surg., 54: 681-685, 1992.

HAMMERMEISTER, K. E.; SETHI, G. K.; HENDERSON, W. G.; OPRIAN, C.; KIM, T.; RAHIMTOOLA, S. A. - Comparison of outcomes in men 11 years after heart-valve replacement with a mechanical valve or bioprosthesis. N. Engl. J. Med., 328: 1289-1296, 1993.

4 PILLAI, R.; SPRIGGINGS, D.; AMARASENA, N. Stentless aortic bioprothesis? The way forwards early experience with the Edwards valve. Ann. Thorac. Surg., 56: 88-91, 1993.
5 THUBRIKAR, M. J.; HECKMAN, J. L.; NOLAN, S. P. High speed cine-radiograph study of aortic valve leaflet motion. J. Heart Valve Dis., 6: 653-661, 1993.

6 VRANDECIC, M. P.; GONTIJO, B. F.; FANTINI, F. A. - Anatomically Complete Heterograft Mitral Valve Substitute: Surgical Technique and Immediate Results. J. Heart Valve Dis., 5: 320-332, 1992.

7 VRANDECIC, M. P.; MOREA, M.; RADEGRAN, K. Clinical use of a new stentless porcine aortic bioprothesis: a Multicenter Study: Cardiology and Cardiac Surgery: Current Topics. New York, Futura Publishing Company, Inc., 1992. p. 223-234.

8 VRANDECIC, M. P.; GONTIJO, B. F.; FANTINI, F. A. - Stentless porcini aortic valve: mid-term clinical follow-up. Heart Surg., 6: 43-56, 1993. 